\title{
Divergence-free vector fields with inverse shadowing
}

\author{
Keonhee Lee ${ }^{1}$ and Manseob Lee ${ }^{2^{*}}$
}

"Correspondence:

Imsds@mokwon.ac.kr

${ }^{2}$ Department of Mathematics,

Mokwon University, Daejeon,

302-729, Korea

Full list of author information is

available at the end of the article

\begin{abstract}
We show that if a divergence-free vector field has the $C^{1}$-stably orbital inverse shadowing property with respect to the class of continuous methods $\mathcal{T}_{d}$, then the vector field is Anosov. The results extend the work of Bessa and Rocha (J. Differ. Equ. 250:3960-3966, 2011).

MSC: $37 \mathrm{C} 10 ; 37 \mathrm{C} 27 ; 37 \mathrm{C} 50$

Keywords: topological stability; inverse shadowing; orbital inverse shadowing; continuous method; Anosov
\end{abstract}

\section{Introduction}

The notion of inverse shadowing property is a dual notion of the shadowing property. It was studied by [1-7]. In fact, Pilyugin [7] showed that every structurally stable diffeomorphism has the inverse shadowing property with respect to the class of continuous methods. In [3], Lee proved that if a diffeomorphism has the $C^{1}$-stably inverse shadowing property with respect to the class of continuous methods $\mathcal{T}_{d}$, then the diffeomorphism is structurally stable. For vector fields, Lee and Lee [5] introduced the notion of inverse shadowing for flows and showed that every expansive flow with the shadowing property has the inverse shadowing property with respect to the class of continuous methods. Lee et al. [6] showed that the $C^{1}$-interior of the set of vector fields with the orbital shadowing property with respect to the class $\mathcal{T}_{d}$ coincides with the set of structurally stable vector fields. From the facts, Lee [4] showed that if a volume-preserving diffeomorphism has the $C^{1}$-stably inverse shadowing property with respect to the class $\mathcal{T}_{d}$, then the diffeomorphism is Anosov. Moreover, if a volume-preserving diffeomorphism has the $C^{1}$-stably orbital inverse shadowing property with respect to the class $\mathcal{T}_{d}$, then the diffeomorphism is Anosov. In this spirit, we study divergence-free vector fields with the inverse, orbital inverse shadowing property with respect to the class $\mathcal{T}_{d}$.

Let us be more detailed. Let $M$ be a closed, connected, and $C^{\infty}$ Riemannian manifold endowed with a volume form $\mu$, and let $\mu$ denote the Lebesgue measure associated to it. Given a $C^{r}(r \geq 1)$ vector field $X: M \rightarrow T M$, the solution of the equation $x^{\prime}=X(x)$ gives rise to a $C^{r}$ flow, $X^{t}$; on the other hand, given a $C^{r}$ flow, we can define a $C^{r-1}$ vector field by considering $X(x)=\left.\frac{d X^{t}(x)}{d t}\right|_{t=0}$. We say that $X$ is divergence-free if its divergence is equal to zero. Note that, by the Liouville formula, a flow $X^{t}$ is volume-preserving if and only if the corresponding vector field $X$ is divergence-free. Let $\mathfrak{X}_{\mu}^{r}(M)$ denote the space of $C^{r}$ divergence-free vector fields, and we consider the usual $C^{r}$ Whitney topology on

\section{黛 Springer}

(2013 Lee and Lee; licensee Springer. This is an Open Access article distributed under the terms of the Creative Commons Attribution License (http://creativecommons.org/licenses/by/2.0), which permits unrestricted use, distribution, and reproduction in any medium, provided the original work is properly cited. 
this space. Let $X \in \mathfrak{X}_{\mu}^{1}(M)$ for any $\delta>0$ and $T>0$. We say that a $(\delta, T)$-pseudo orbit of $X \in \mathfrak{X}_{\mu}^{1}(M)$ if

$$
d\left(X^{t_{i}}\left(x_{i}\right), x_{i+1}\right)<\delta
$$

for any $t_{i} \geq T, i \in \mathbb{Z}$. A mapping $\Psi: \mathbb{R} \times M \rightarrow M$ is called a $(\delta, T)$-method for $X$ if for any $x \in M$, the map $\Psi_{x}: \mathbb{R} \rightarrow M$ defined by

$$
\Psi_{x}(t)=\Psi(t, x), \quad t \in \mathbb{R},
$$

is a $(\delta, T)$-pseudo orbit of $X . \Psi$ is said to be complete if $\Psi(0, x)=x$ for any $x \in M$. Then a $(\delta, T)$-method for $X$ can be considered a family of $(\delta, T)$-pseudo orbits of $X$. A method $\Psi$ for $X$ is said to be continuous if the map $\Psi^{\prime}: M \rightarrow M^{\mathbb{R}}$, given by $\Psi^{\prime}(x)(t)=\Psi(t, x)$ for $x \in M$ and $t \in \mathbb{R}$, is continuous under the compact open topology on $M^{\mathbb{R}}$, where $M^{\mathbb{R}}$ denotes the set of all functions from $\mathbb{R}$ to $M$. The set of all complete $(\delta, 1)$-methods [resp. complete continuous $(\delta, 1)$-methods] for $X \in \mathfrak{X}_{\mu}^{1}(M)$ will be denoted by $\mathcal{T}_{a}(\delta, X)\left[\operatorname{resp} . \mathcal{T}_{c}(\delta, X)\right.$ ]. We can see that if $Y$ is another vector field which is sufficiently close to $X$ in the $C^{0}$ topology, then it induces a complete continuous method for $X$. Let $\mathcal{T}_{h}(\delta, X)$ [resp. $\mathcal{T}_{d}(\delta, X]$ be the set of all complete continuous $(\delta, 1)$ methods for $X$ which are induced by $C^{1}$ vector fields $Y$ with $d_{0}(X, Y)<\delta$ [resp. $d_{1}(X, Y)<\delta$ ], where $d_{0}$ is the $C^{0}$ metric on $\mathfrak{X}_{\mu}^{1}(M)$ such that

$$
d_{0}(X, Y)=\sup _{x \in M}\{\|X(x)-Y(x)\|\}
$$

and $d_{1}$ is the $C^{1}$ metric on $\mathfrak{X}_{\mu}^{1}(M)$ such that

$$
d_{1}(X, Y)=d_{0}(X, Y)+\sup _{x \in M}\|D X(x)-D Y(x)\| .
$$

We say that a vector field $X$ has the inverse shadowing property with respect to the class $\mathcal{T}_{\alpha}(\alpha=a, c, h, d)$ if for any $\epsilon>0$, there is $\delta>0$ such that for any $(\delta, T)$-method $\Psi \in \mathcal{T}_{\alpha}(\delta, X)$ and any point $x \in M$, there are $y \in M$ and an increasing homeomorphism $h: \mathbb{R} \rightarrow \mathbb{R}$ with $h(0)=0$ such that

$$
d\left(X^{h(t)}(x), \Psi(t, y)\right)<\epsilon, \quad t \in \mathbb{R} .
$$

We denote by $\mathcal{I S}_{\mu, \alpha}(M)$ the set of divergence-free vector fields on $M$ with the inverse shadowing property with respect to the class $\mathcal{T}_{\alpha}$, where $\alpha=a, c, h, d$. Let int $\mathcal{I} \mathcal{S}_{\mu, \alpha}(M)$ be the $C^{1}$-interior of the set of divergence-free vector fields on $M$ with the inverse shadowing property with respect to the class $\mathcal{T}_{\alpha}$, where $\alpha=a, c, h, d$.

We say that $X \in \mathfrak{X}_{\mu}^{1}(M)$ has the $C^{1}$-stably inverse shadowing property with respect to the class $\mathcal{T}_{\alpha}$ if there is a $C^{1}$-neighborhood $\mathcal{U}(X) \subset \mathfrak{X}_{\mu}^{1}(M)$ of $X$ such that for any $Y \in \mathcal{U}(X)$, $Y$ has the inverse shadowing property with respect to the class $\mathcal{T}_{d}$, where $\alpha=a, c, h, d$.

Remark 1.1 Let $X \in \mathfrak{X}_{\mu}^{1}(M)$. Then $\mathcal{I S}_{\mu, a}(M) \subset \mathcal{I} \mathcal{S}_{\mu, c}(M) \subset \mathcal{I} \mathcal{S}_{\mu, h}(M) \subset \mathcal{I} \mathcal{S}_{\mu, d}(M)$, where $\alpha=a, c, h, d$. 
We say that $X \in \mathfrak{X}_{\mu}^{1}(M)$ has the orbital inverse shadowing property with respect to the class $\mathcal{T}_{d}$ if for any $\epsilon>0$, there is $\delta>0$ such that for any $(\delta, 1)$-method $\Psi \in \mathcal{T}_{d}(\delta, X)$ and any point $x \in M$, there is $y \in M$ such that

$$
d_{H}\left(\overline{\operatorname{Orb}_{X}(x)}, \overline{\operatorname{Orb}(y, \Psi)}\right)<\epsilon,
$$

where $\operatorname{Orb}(y, \Psi)=\{\Psi(t, y): t \in \mathbb{R}\}$. Note that if $X$ has the inverse shadowing property with respect to the class $\mathcal{T}_{d}$, then $X$ has the orbital shadowing property with respect to the class $\mathcal{T}_{d}$. But the converse is not true. Indeed, an irrational rotation map does not have the inverse shadowing property. And the map has the orbital shadowing property. Let int $\mathcal{O I S}_{\mu, \alpha}(M)$ be the $C^{1}$-interior of the set of divergence-free vector fields on $M$ with the orbital inverse shadowing property with respect to the class $\mathcal{T}_{\alpha}$, where $\alpha=a, c, h, d$.

Let $\Lambda$ be a closed $X_{t}$-invariant set. We say that $\Lambda$ is hyperbolic if there are constants $C>0, \lambda>0$ and a continuous splitting $T_{\Lambda} M=E^{s} \oplus\langle X(x)\rangle \oplus E^{u}$ such that

$$
\left\|\left.D X^{t}\right|_{E^{s}(x)}\right\| \leq C e^{-\lambda t} \text { and }\left\|\left.D X^{-t}\right|_{E^{u}(x)}\right\| \leq C e^{-\lambda t}
$$

for any $x \in \Lambda$ and $t>0$. If $\Lambda=M$, then $X$ is called Anosov.

Given a vector field $X$, we denote by $P O(X)$ the set of closed orbits of $X$ and by $\operatorname{Sing}(X)$ the set of singularities of $X$, i.e., those points $x \in M$ such that $X(x)=\overrightarrow{0}$. Denote by $\operatorname{Crit}(X)=$ $\operatorname{Sing}(X) \cup P O(X)$. Let $R:=M \backslash \operatorname{Sing}(X)$ be the set of regular points. We assume that the exponential map $\exp _{p}: T_{p} M(1) \rightarrow M$ is well defined for all $p \in M$, where $T_{p} M(1)=\{v \in$ $\left.T_{p} M:\|v\| \leq 1\right\}$. Given $x \in R$, we consider its normal bundle $N_{x}=\langle X(x)\rangle^{\perp} \subset T_{x} M$, and let $N_{x}(r)$ be the $r$-ball in $N_{x}$. Let $\mathcal{N}_{x, r}=\exp _{x}\left(N_{x}(r)\right)$. For any $x \in R$ and $t \in \mathbb{R}$, there are $r>0$ and a $C^{1}$ map $\tau: \mathcal{N}_{x, r} \rightarrow \mathbb{R}$ with $\tau(x)=t$ such that $X^{\tau(y)}(y) \in \mathcal{N}_{X^{t}(x), 1}$ for any $y \in \mathcal{N}_{x, r}$. We say $\tau(y)$ is the first return time of $y$. Then we define the Poincarè map $f$ by

$$
\begin{aligned}
& f: \mathcal{N}_{X, r} \rightarrow \mathcal{N}_{X^{t}(x), 1}, \\
& y \mapsto f(y)=X^{\tau(y)}(y) .
\end{aligned}
$$

Let $\mathcal{N}=\bigcup_{x \in R} \mathcal{N}_{x}$ be the normal bundle based on $R$. We can define the associated linear Poincaréflow by

$$
P_{X}^{t}(x):=\Pi_{X^{t}(x)} \circ D X^{t}(x)
$$

where $\Pi_{X^{t}(x)}: T_{X^{t}(x)} M \rightarrow N_{X^{t}(x)}$ is the projection along the direction of $X\left(X^{t}(x)\right)$. We say that a vector field $X \in \mathfrak{X}_{\mu}^{1}(M)$ is topologically stable if for any $\epsilon>0$, there is $\delta>0$ such that for any $Y \in \mathfrak{X}_{\mu}^{1}(M)$ with $d_{0}(X, Y)<\delta$, there is a semi-conjugacy $(h, \tau)$ from $Y$ to $X$ satisfying $d(h(x), x)<\epsilon$ for all $x \in M$.

In [8], the authors proved that if a vector field is in the $C^{1}$-interior of the set of topologically stable vector fields (not divergence-free vector fields), then $X$ satisfies Axiom A and the strong transversality condition. Robinson [9] proved that if a vector field satisfies Axiom A and the strong transversality condition, then the vector field is structurally stable. For divergence-free vector fields, Bessa and Rocha [10] showed that if a divergence-free vector field is in the $C^{1}$-interior of the set of topological stable vector fields $X \in \mathfrak{X}_{\mu}^{1}(M)$, then $X$ is Anosov. 
Remark 1.2 We have the following implication: topological stability $\Rightarrow$ inverse shadowing property with respect to the continuous method $\mathcal{T}_{d} \Rightarrow$ orbital inverse shadowing property with respect to the continuous method $\mathcal{T}_{d}$.

From the above remark, we know that our result is a slight generalization of the main theorem in [10]. In this paper, we omit the phrase 'with respect to the class $\mathcal{T}_{d}$ ' for simplicity. So, we say that $X$ has the inverse, orbital inverse shadowing property means that $X$ has the inverse, orbital inverse shadowing property with respect to the class $\mathcal{T}_{d}$. Note that if $X \in \operatorname{int} \mathcal{I S}_{\mu, \alpha}^{1}(M)$ or $X \in \operatorname{int} \mathcal{O I S}_{\mu, \alpha}^{1}(M)$, then it means that $X$ has the $C^{1}$-stably inverse, orbital inverse shadowing property with respect to the class $\mathcal{T}_{\alpha}, \alpha=a, c, h, d$. The following is the main result of this paper.

Theorem 1.3 Let $X \in \mathfrak{X}_{\mu}^{1}(M)$. Then

$$
\operatorname{int} \mathcal{I S}_{\mu, d}^{1}(M)=\operatorname{int} \mathcal{O I S}_{\mu}^{1}(M)=\mathcal{A}_{\mu}^{1}(M)
$$

where $\mathcal{A}_{\mu}^{1}(M)$ is the set of divergence-free Anosov vector fields.

\section{Proof of Theorem 1.3}

Let $M$ be as before, and let $X \in \mathfrak{X}_{\mu}^{1}(M)$. The perturbations (Lemma 2.1) for volumepreserving vector fields allows to realize them as perturbations of a fixed volumepreserving flow. Fix $X \in \mathfrak{X}_{\mu}^{1}(M)$ and $\tau>0$. A one-parameter area-preserving linear family $\left\{A_{t}\right\}_{t \in \mathbb{R}}$ associated to $\left\{X^{t}(p) ; t \in[0, \tau]\right\}$ is defined as follows:

- $A_{t}: N_{p} \rightarrow N_{p}$ is a linear map for all $t \in \mathbb{R}$,

- $A_{t}=i d$ for all $t \leq 0$ and $A_{t}=A_{\tau}$ for all $t \geq \tau$,

- $A_{t} \in S L(n, \mathbb{R})$, and

- the family $A_{t}$ is $C^{\infty}$ on the parameter $t$.

The following result, proved in [11, Lemma 3.2], is now stated for $X \in \mathfrak{X}_{\mu}^{1}(M)$ instead of $X \in \mathfrak{X}_{\mu}^{4}(M)$ because of the improved smooth $C^{1}$ pasting lemma proved in [12, Lemma 5.2].

Lemma 2.1 Given $\epsilon>0$ and a vector field $X \in \mathfrak{X}_{\mu}^{1}(M)$, there exists $\xi_{0}=\xi_{0}(\epsilon, X)$ such that for all $\tau \in[1,2]$, for any periodic point $p$ of period greater than 2 , for any sufficiently small flowbox $\mathcal{T}$ of $\left\{X_{t}(p) ; t \in[0, \tau]\right\}$ and for any one-parameter linear family $\left\{A_{t}\right\}_{t \in[0, \tau]}$ such that $\left\|A_{t}^{\prime} A_{t}^{-1}\right\|<\xi_{0}$ for all $t \in[0, \tau]$, there exists $Y \in \mathfrak{X}_{\mu}^{1}(M)$ satisfying the following properties:

(a) $Y$ is $\epsilon$ - $C^{1}$-close to $X$;

(b) $Y^{t}(p)=X^{t}(p)$ for all $t \in \mathbb{R}$;

(c) $P_{Y}^{\tau}(p)=P_{X}^{\tau}(p) \circ A_{\tau}$, and

(d) $\left.\left.Y\right|_{\mathcal{T}^{c}} \equiv X\right|_{\mathcal{T}^{c}}$.

Remark 2.2 Let $X \in \mathfrak{X}_{\mu}^{1}(M)$. By Zuppa's theorem [13], we can find $Y C^{1}$-close to $X$ such that $Y \in \mathfrak{X}_{\mu}^{\infty}(M), Y^{\pi}(p)=p$ and $P_{Y}^{\pi}(p)$ has an eigenvalue $\lambda$ with $|\lambda|=1$.

A divergence-free vector field $X$ is a divergence-free star vector field if there exists a $C^{1}$ neighborhood $\mathcal{U}(X)$ of $X$ in $\mathfrak{X}_{\mu}^{1}(M)$ such that if $Y \in \mathcal{U}(X)$, then every point in $\operatorname{Crit}(Y)$ is hyperbolic. The set of divergence-free star vector fields is denoted by $\mathcal{G}_{\mu}^{1}(M)$. Then we get the following theorem. 
Theorem 2.3 [14, Theorem 1.1] If $X \in \mathcal{G}_{\mu}^{1}(M)$, then $\operatorname{Sing}(X)=\emptyset$ and $X$ is Anosov.

Thus, to prove Theorem 1.3, it is enough to show that if $X$ has the inverse shadowing property, or the orbital inverse shadowing property, then $X \in \mathcal{G}_{\mu}^{1}(M)$.

Proposition 2.4 Let $X \in \operatorname{int} \mathcal{I S}_{\mu, d}^{1}(M)$. Then $X \in \mathcal{G}_{\mu}^{1}(M)$.

Proof Suppose that $X \in \mathfrak{X}_{\mu}^{1}(M)$ has the $C^{1}$-stably inverse shadowing property. Then there is a $C^{1}$-neighborhood $\mathcal{U}(X)$ of $X$ such that for any $Y \in \mathcal{U}(X), Y$ has the inverse shadowing property. Let $p \in \gamma \in P O(X)$ with $X^{\pi}(p)=p$ and $U_{p}$ be a small neighborhood of $p \in M$. We will derive a contradiction. Assume that there is an eigenvalue $\lambda$ of $P_{X}^{\pi}(p)$ such that $|\lambda|=1$. By Remark 2.2, there is $Y \in \mathcal{U}(X)$ such that $Y \in \mathfrak{X}_{\mu}^{\infty}(M), Y_{Y}^{\pi}(p)=p$ and $P_{Y}^{\pi}(p)$ has an eigenvalue $\lambda$ with $|\lambda|=1$. Using Moser's theorem (see [15]), there is a smooth conservative change of coordinates $\varphi_{p}: U_{p} \rightarrow T_{p} M$ such that $\varphi_{p}(p)=\overrightarrow{0}$. Let $f: \varphi_{p}^{-1}\left(N_{p}\right) \rightarrow \mathcal{N}_{p}$ be the Poincarè map associated to $Y^{t}$, and let $\mathcal{V}$ be a $C^{1}$-neighborhood of $f$. Here, $\mathcal{N}_{p}$ is the Poincarè section through $p$. By Lemma 2.1, we can find a small flowbox $\mathcal{T}$ of $Y^{\left[0, t_{0}\right]}$, $0<t_{0}<\pi$, and there are $Z \in \mathcal{U}_{0}(Y) \subset \mathcal{U}(X), g \in \mathcal{V}$ and $\alpha>0$ such that

(a) $Z^{t}=Y^{t}$ for all $t \in \mathbb{R}$,

(b) $P_{Z}^{t_{0}}(p)=P_{Y}^{t_{0}}(p)$

(c) $\left.Z\right|_{\mathcal{T}^{c}}=\left.Y\right|_{\mathcal{T}^{c}}$,

(d) $g(x)=\varphi_{p}^{-1} \circ P_{Y}^{\pi}(p) \circ \varphi_{p}(x)$ for all $x \in B_{\alpha}(p) \cap \varphi_{p}^{-1}\left(N_{p}\right)$, and

(e) $g(x)=f(x)$ for all $x \notin B_{4 \alpha}(p) \cap \varphi_{p}^{-1}\left(N_{p}\right)$.

Then $P_{Z}^{\pi}(p)$ has an eigenvalue $\lambda$ with $|\lambda|=1$. For $0<\epsilon<\alpha / 8$, let $0<\delta<\epsilon$ be as in the definition of the inverse shadowing property of $Z^{t}$.

Take a linear map $A_{t}: N_{p} \rightarrow N_{p}$ for all $t \in \mathbb{R}$ such that if $\left\|P_{Z}^{\pi}(p) \circ A_{t_{0}}-P_{Z}^{\pi}(p)\right\|<\delta_{0}$, then $d_{1}(Z, W)<\delta$, and $P_{Z}^{\pi}(p) \circ A_{t_{0}}$ is a hyperbolic linear Poincarè flow. Set $P_{W}^{t}(p)=P_{Z}^{\pi}(p) \circ A_{t_{0}}$. Then $W^{t} \in \mathcal{T}_{d}(Z)$, and $p \in \gamma$ is a periodic point of $W^{t}$. Since $Z \in \mathcal{U}(X)$ for any $x \in M$, there exist $y \in M$ and an increasing homeomorphism $h: \mathbb{R} \rightarrow \mathbb{R}$ with $h(0)=0$ such that $d\left(Z^{h(t)}(x), W^{t}(y)\right)<\epsilon$ for all $t \in \mathbb{R}$.

First, we assume that $\lambda=1$ (the other case is similar). Then we can choose a vector $v$ associated to $\lambda$ such that $\|v\|<\alpha / 4$. Since $\varphi_{p}^{-1}(v) \in \varphi_{p}^{-1}\left(N_{p}\right) \backslash\{p\}$,

$$
g\left(\varphi_{p}^{-1}(v)\right)=\varphi_{p}^{-1} \circ P_{Y}^{\pi}(p) \circ \varphi_{p}\left(\varphi_{p}^{-1}(p)\right)=\varphi_{p}^{-1} \circ P_{Y}^{\pi}(p)(v)=\varphi_{p}^{-1}(v) .
$$

Then we can take $z \in \varphi_{p}^{-1}\left(N_{p}\right)$ such that

$$
p \notin B_{\epsilon}\left\{g_{1}^{i}(z): i \in \mathbb{Z}\right\} \subset B_{\alpha / 2}(p) \cap \varphi_{p}^{-1}\left(N_{p}\right),
$$

where $g_{1}$ is the Poincarè map associated to $W^{t}$. Since $P_{W}^{t}(p)$ is a hyperbolic linear Poincarè flow, we can see that

$$
d\left(g^{i}(p), g_{1}^{i}(z)\right)>\epsilon
$$

for some $i \in \mathbb{Z}$. Thus

$$
d\left(Z^{h(t)}(p), W^{t}(z)\right)>\epsilon
$$


for some $t \in \mathbb{R}$. This is a contradiction by the fact that $Z$ has the inverse shadowing property.

Finally, we assume that $\lambda$ is complex. By [10, Lemma 3.2], there is $Z \in \mathcal{U}(X)$ such that $P_{Z}^{\pi}(p)$ is a rational rotation. Then there is $l>0$ such that $P_{Z}^{l+\pi}(p)$ is the identity. As in the previous argument, we get a contradiction.

Proposition 2.5 Let $X \in \operatorname{int} \mathcal{O I S}_{\mu, d}^{1}(M)$. Then $X \in \mathcal{G}_{\mu}^{1}(M)$.

Proof Suppose that $X$ has the $C^{1}$-stably orbital inverse shadowing property. Then there is a $C^{1}$-neighborhood $\mathcal{U}(X)$ of $X$ such that for any $Y \in \mathcal{U}(X), Y$ has the orbital inverse shadowing property. Let $p \in \gamma \in P O(X)$ with $X^{\pi}(p)=p$ and $U_{p}$ be a small neighborhood of $p \in M$. We will derive a contradiction. Assume that there is an eigenvalue $\lambda$ of $P_{X}^{\pi}(p)$ such that $|\lambda|=1$. As in the proof of Proposition 2.4, we get a hyperbolic linear Poincarè flow $P_{W}^{t}(p)$, and $W^{t} \in \mathcal{T}_{d}(Z)$, and $p \in \gamma$ is a periodic point of $W^{t}$. Since $Z \in \mathcal{U}(X)$ for any $x \in M$, there exist $y \in M$ such that

$$
d_{H}\left(\overline{\mathcal{O}\left(x, Z^{t}\right)}, \overline{\mathcal{O}\left(y, W^{t}\right)}\right)<\epsilon
$$

for all $t \in \mathbb{R}$. Take $t^{\prime}=\min \left\{|t|: W^{t}(y) \in \varphi_{p}^{-1}\left(N_{p}\right)\right\}$, and let $w=W^{t^{\prime}}(y) \in \varphi_{p}^{-1}\left(N_{p}\right)$.

If $\lambda \in \mathbb{R}$ or $\lambda \in \mathbb{C}$, then as in the proof of Lemma 2.4, we get a contradiction. Indeed, since $P_{W}^{t}(p)$ is a hyperbolic linear Poincarè flow, there is $j>0$ such that $g_{1}^{j}(w) \notin B_{\alpha / 4}(p) \cap \varphi_{p}^{-1}\left(N_{p}\right)$. Thus

$$
d_{H}\left(\overline{\mathcal{O}\left(x, Z^{t}\right)}, \overline{\mathcal{O}\left(w, W^{t}\right)}\right)>\epsilon
$$

for some $t \in \mathbb{R}$. Since $Z \in \mathcal{U}(X)$, this is a contradiction.

End of the proof of Theorem 1.3 By Proposition 2.4 and Proposition 2.5, we have $X \in$ $\mathcal{G}_{\mu}^{1}(M)$. Thus by Theorem 2.3, we get $\operatorname{Sin} g(X)=\emptyset$ and $X$ is Anosov.

From the result of [10], we get the following corollary.

Corollary 2.6 Let $X \in \mathfrak{X}_{\mu}^{1}(M)$. Then

$$
\operatorname{int} \mathcal{T} \mathcal{S}_{\mu}^{1}(M)=\operatorname{int} \mathcal{I} \mathcal{S}_{\mu, d}^{1}(M)=\operatorname{int} \mathcal{O} \mathcal{I} \mathcal{S}_{\mu}^{1}(M)=\operatorname{int} \mathcal{A}_{\mu}^{1}(M),
$$

where int $\mathcal{T S}_{\mu}^{1}(M)$ is the $C^{1}$-interior of the set of divergence-free vector fields on $M$ which are topologically stable.

Competing interests

The authors declare that they have no competing interests.

Authors' contributions

All authors read and approved the final manuscript. 


\section{Acknowledgements}

The first author is supported by the National Research Foundation (NRF) of Korea funded by the Korean Government (No. 2011-0015193). The second author is supported by the Basic Science Research Program through the National Research Foundation of Korea (NRF) funded by the Ministry of Education, Science and Technology, Korea (No. 2011-0007649).

Received: 4 March 2013 Accepted: 23 October 2013 Published: 21 Nov 2013

\section{References}

1. Corless, R, Pilugin, SY: Approximate and real trajectories for generic dynamical systems. J. Math. Anal. Appl. 189 409-423 (1995)

2. Han, Y, Lee, K: Inverse shadowing for structurally stable for flows. Dyn. Syst. 19, 371-388 (2004)

3. Lee, K: Continuous inverse shadowing and hyperbolicity. Bull. Aust. Math. Soc. 67, 15-26 (2003)

4. Lee, M: Volume-preserving diffeomorphisms with inverse shadowing. J. Inequal. Appl. 2012(275), 1-9 (2012)

5. Lee, K, Lee, Z: Inverse shadowing for expansive flows. Bull. Korean Math. Soc. 40, 703-713 (2003)

6. Lee, K, Lee, Z, Zhang, Y: Structural stability of vector fields with orbital inverse shadowing. J. Korean Math. Soc. 45, 1505-1521 (2008)

7. Pilygin, SY: Inverse shadowing by continuous methods Discrete Contin. Dyn. Syst. 8, 29-38 (2002)

8. Moriyasu, K, Sakai, K, Sumi, N: Vector fields with topological stability. Trans. Am. Math. Soc. 353, 3391-3408 (2001)

9. Robinson, C: Structural stability of vector fields. Ann. Math. 99, 154-175 (1974)

10. Besa, M, Rocha, J: Topological stability for conservative systems. J. Differ. Equ. 250, 3960-3966 (2011)

11. Bessa, M, Rocha, J: On C C-robust transitivity of volume-preserving flows. J. Differ. Equ. 245(11), 3127-3143 (2008)

12. Bessa, M, Rocha, J: Contributions to the geometric and ergodic theory of conservative flows. Ergod. Theory Dyn. Syst. (2012, at press)

13. Zuppa, C: Regularisation $C^{\infty}$ des champs vectoriels qui préservent lélément de volume. Bol. Soc. Bras. Mat. 10, 51-56 (1979)

14. Ferreira, C: Stability properties of divergence-free vector fields. Dyn. Syst. 27, 223-238 (2012)

15. Moser, J: On the volume elements on a manifold. Trans. Am. Math. Soc. 120, 286-294 (1965)

10.1186/1687-1847-2013-337

Cite this article as: Lee and Lee: Divergence-free vector fields with inverse shadowing. Advances in Difference Equations 2013, 2013:337

\section{Submit your manuscript to a SpringerOpen ${ }^{\circ}$ journal and benefit from:}

- Convenient online submission

- Rigorous peer review

- Immediate publication on acceptance

- Open access: articles freely available online

- High visibility within the field

- Retaining the copyright to your article 